Research Article

\title{
An Averaging Principle for Mckean-Vlasov-Type Caputo Fractional Stochastic Differential Equations
}

\author{
Weifeng Wang $\mathbb{D},{ }^{1}$ Lei Yan $\mathbb{D}^{2},{ }^{2}$ Junhao Hu $\mathbb{D},{ }^{1}$ and Zhongkai Guo $\mathbb{D}^{1}$ \\ ${ }^{1}$ School of Mathematics and Statistics, South-Central University for Nationalities, Wuhan 430074, China \\ ${ }^{2}$ School of General Quality Education, Wuchang University of Technology, Wuhan 430223, China \\ Correspondence should be addressed to Zhongkai Guo; zkguo@scuec.edu.cn
}

Received 13 May 2021; Accepted 30 June 2021; Published 16 July 2021

Academic Editor: Lifeng Wu

Copyright ( 92021 Weifeng Wang et al. This is an open access article distributed under the Creative Commons Attribution License, which permits unrestricted use, distribution, and reproduction in any medium, provided the original work is properly cited.

In this paper, we want to establish an averaging principle for Mckean-Vlasov-type Caputo fractional stochastic differential equations with Brownian motion. Compared with the classic averaging condition for stochastic differential equation, we propose a new averaging condition and obtain the averaging convergence results for Mckean-Vlasov-type Caputo fractional stochastic differential equations.

\section{Introduction}

For complex systems, we usually want to locate an effective simplified model to approximate the original complex system or extract the main dynamical behavior of the original system. Based on these ideas, a lot of effective methods have been generated in dynamical systems, such as invariant manifolds, averaging principle, and homogenization principle. These effective methods have now been extended to deal with stochastic systems, such as stochastic invariant manifolds see $[1,2]$ and stochastic averaging principle, see [3-9].

Currently, the problem of averaging for stochastic differential equations have received a lot of attention and various types of stochastic differential equations have been studied, see $[4,6,7,10-12]$. However, there are no relevant results of averaging principle for distribution dependent-type stochastic differential equations which we will consider in this paper.

On the contrary, the problem of averaging for stochastic fractional order differential equations have received a lot of attention in recent years, and some results [13] have been obtained under averaging condition consistence with the classic case (see $[4,5,14])$. Noting that the fractional order derivative is a nonlocal operator, therefore, the fractional order differential equation is more effective for describing certain phenomena in the real world (see [15-17]). Current research studies on stochastic fractional order differential equations mainly focused on the existence and uniqueness of the solutions, with fewer results from the dynamical system perspective.

Based on the above discussion, we shall study the averaging principle for the following Mckean-Vlasov-type Caputo fractional stochastic differential equations:

$$
\left\{\begin{array}{l}
D_{t}^{\alpha} X_{t}=f\left(t, X_{t}, \mu_{t}\right) \mathrm{d} t+g\left(t, X_{t}, \mu_{t}\right) \mathrm{d} B_{t}, \quad t \geq 0, \\
\mu(t)=\text { probability distribution of } X_{t}, \\
X_{0}=x_{0} \in L^{2}(\Omega, H),
\end{array}\right.
$$

where $\alpha \in(1 / 2,1]$ and $B_{t}$ is a scalar Brownian motion. Nonlinear terms $f$ and $g$ are $H$-valued functions defined on $R^{+} \times H \times M_{\gamma^{2}}(H)$, and $M_{\gamma^{2}}(H)$ denotes a proper subset of probability measure on $H$. If the terms $f$ and $g$ do not depend on the probability distribution $\mu(t)$ of the process $X$ at time $t$, such equations have been studied by [13] and other authors. If $\alpha=1$, the equation becomes a classical Mckean-Vlasov-type stochastic differential equations which have been considered by many authors with different approaches (see [18-20]). In this paper, we just focused on $\alpha \in(1 / 2,1)$, and more details can be seen in Section 2 .

The paper is structured as follows. We introduce some notation and assumptions in Section 2. The existence and unique solution for distribution dependent fractional 
stochastic differential equations will be discussed in Section 3. An averaging principle for the above equation is established in Section 4.

\section{Preliminaries}

First, we introduce some notation. Let $C(H)$ be the space of continuous functions on $H$. Let $\mathscr{B}(H)$ be the Borel $\sigma$-algebra of subsets of $H . M(H)$ is the space of probability measures on $\mathscr{B}(H)$ and carries the usual topology of weak convergence. $(\mu, \phi)$ denotes $\int_{H} \phi(x) \mu(\mathrm{d} x)$. Let $\gamma(x)=1+$ $|x|, \forall x \in H$, and then, define the Banach space

$$
C_{\rho}(H)=\left\{\phi \in C(H):\|\phi\|_{C_{\rho}} \equiv \sup _{x \in H} \frac{|\phi(x)|}{\gamma^{2}(x)}+\sup _{x \neq y} \frac{|\phi(x)-\phi(y)|}{|x-y|}<\infty\right\} .
$$

For any $p \geq 1$, let $M_{\gamma^{p}}^{s}(H)$ denote the Banach space of signed measures $m$ on $H$, and $\|\mu\|_{\gamma^{p}} \equiv \int_{H} \gamma^{p}|m|(\mathrm{d} x)<\infty$. $|m|=m^{+}+m^{-}$and $m=m^{+}-m^{-}$are the Jordan decomposition of $m \cdot M_{\gamma^{2}}(H)=M_{\gamma^{p}}^{s}(H) \cap M(H)$ is the set of probability measures on $\mathscr{B}(H)$, and there exists second moments. Define the following metric:

$$
\rho(\mu, \nu)=\sup \left\{(\phi, \mu-\nu):\|\phi\|_{\rho}=\sup _{x \in H} \frac{|\phi(x)|}{\gamma^{2}(x)}+\sup _{x \neq y} \frac{|\phi(x)-\phi(y)|}{|x-y|} \leq 1\right\}
$$

Then, $\left(M_{\gamma^{2}}(H), \rho\right)$ is a complete metric space. Let $C\left([0, T],\left(M_{\gamma^{2}}(H), \rho\right)\right)$ be the complete metric space of continuous functions from $[0, T]$ to $\left(M_{\gamma^{2}}(H), \rho\right)$ with the following metric:

$$
D_{T}(\mu, \nu)=\sup _{t \in[0, T]} \rho(\mu(t), \nu(t)), \quad \text { for } \nu, \mu \in C\left([0, T], M_{\gamma^{2}}(H), \rho\right) .
$$

More details can be seen in [18].

In order to obtain the existence and uniqueness of the solution of (1), we introduce the following conditions.

(i) H1 (Lipschitz condition): for all $x, y \in H$ and $t \in[0, T], \mu \in C\left([0, T], M_{\gamma^{2}}(H), \rho\right)$, and there exists a bounded function $k_{1}(t)>0$, such that

$$
\begin{aligned}
& |f(t, x, \mu)-f(t, y, \nu)|^{2}+|g(t, x, \mu)-g(t, y, \nu)|^{2} \\
& \quad \leq k_{1}(t)\left(|x-y|^{2}+\rho^{2}(\mu, \nu)\right) .
\end{aligned}
$$

(ii) $\mathrm{H} 2$ (growth condition): for all $(x, t) \in H \times[0, T]$, there exists a bounded function $k_{2}(t)>0$ such that

$$
|f(t, x, \mu)|^{2}+|g(t, x, \mu)|^{2} \leq k_{2}(t)\left(1+|x|^{2}\right) .
$$

In this paper, we assume there existence of a constant $k$ such that $\max \left\{k_{1}(t), k_{2}(t)\right\} \leq k$.

First, we give an important lemma, which is a type of promotion form of Gronwall's inequality with singular kernels.

Lemma 1 (see $[21,22]$ ). Suppose $b \geq 0, \beta>0$, and $a(t)$ is $a$ nonnegative function locally integrable on $0 \leq t<T$ (some
$T \leq+\infty)$, and suppose $u(t)$ is nonnegative and locally integrable on $0 \leq t<T$ with

$$
u(t) \leq a(t)+b \int_{0}^{t}(t-s)^{\beta-1} u(s) \mathrm{d} s,
$$

on this interval. Then,

$$
u(t) \leq a(t)+\int_{0}^{t}\left[\sum_{n=1}^{\infty} \frac{(b \Gamma(\beta))^{n}}{\Gamma(n \beta)}(t-s)^{n \beta-1} a(s)\right] \mathrm{d} s, \quad 0 \leq t<T .
$$

\section{Existence and Uniqueness}

Consider the integral form of equation (1):

$$
\begin{aligned}
X_{t}= & x_{0}+\frac{1}{\Gamma(\alpha)} \int_{0}^{t}(t-s)^{\alpha-1} f\left(s, X_{s}, \mu_{t}\right) \mathrm{d} s \\
& +\frac{1}{\Gamma(\alpha)} \int_{0}^{t}(t-s)^{\alpha-1} g\left(t, X_{s}, \mu_{t}\right) \mathrm{d} B_{t} .
\end{aligned}
$$

Under the assumptions of $\mathrm{H} 1$ and $\mathrm{H} 2$, we will prove the existence and uniqueness of solution for the above equation.

Definition 1. An $\mathscr{F}_{t}$-adapted stochastic process $X_{t}$ with law $L\left(X_{t}\right)=\mu(t)$ is called a solution of (1) if $X_{t}$ is continuous, and for $\forall t \in[0, T]$ with $X_{0}=x_{0}$,

$$
\begin{aligned}
X_{t}= & x_{0}+\frac{1}{\Gamma(\alpha)} \int_{0}^{t}(t-s)^{\alpha-1} f\left(s, X_{s}, \mu_{t}\right) \mathrm{d} s \\
& +\frac{1}{\Gamma(\alpha)} \int_{0}^{t}(t-s)^{\alpha-1} g\left(t, X_{s}, \mu_{t}\right) \mathrm{d} B_{t}, \mathbb{P}-\text { a.s. }
\end{aligned}
$$

Theorem 1. Assume that $H 1$ and $H 2$ hold; then, for $\forall x_{0} \in L^{2}(\Omega, H)$, equation (10) has a unique solution 
$X_{t} \in C\left([0, T] ; L^{2}(\Omega, H)\right)$ with the associate probability distribution $\mu_{t}=L\left(X_{t}\right), t \in[0, T]$ belonging to $C([0, T]$, $\left.\left(M_{\gamma}^{2}(X), \rho\right)\right)$, such that

$$
\sup _{0 \leq t \leq T} E\left|X_{t}\right|^{2}<\infty
$$

We will proof the theorem by several steps.

(i) First, we prove that $X_{t} \in L^{\infty}\left([0, T], L^{2}(\Omega ; H)\right)$ for $\forall \mu \in C\left([0, T],\left(M_{\gamma^{2}}(H), \rho\right)\right)$. Using the following inequality,

$$
|a+b+c|^{2} \leq 3\left(|a|^{2}+|b|^{2}+|c|^{2}\right)
$$

We see that

$$
\begin{aligned}
E|X(t)|^{2} \leq & 3 E\left|x_{0}\right|^{2}+3 E\left|\frac{1}{\Gamma(\alpha)} \int_{0}^{t}(t-s)^{\alpha-1} f\left(s, X_{s}, \mu_{s}\right) \mathrm{d} s\right|^{2} \\
& +3 E\left|\frac{1}{\Gamma(\alpha)} \int_{0}^{t}(t-s)^{\alpha-1} g\left(t, X_{s}, \mu_{s}\right) \mathrm{d} B_{t}\right|^{2} \\
:= & 3 I_{1}+3 I_{2}+3 I_{3} .
\end{aligned}
$$

For $I_{2}$, applying Cauchy-Schwarz's inequality and $\mathrm{H} 2$, it follows

$$
\begin{aligned}
I_{2} & \leq \frac{T k}{\Gamma(\alpha)^{2}} \int_{0}^{t}(t-s)^{2 \alpha-2}\left(1+E|X(s)|^{2}+\left\|\mu_{t}\right\|_{\gamma}^{2}\right) \mathrm{d} s \\
& \leq \frac{T k}{\Gamma(\alpha)^{2}}\left[\frac{t^{2 \alpha-1}}{2 \alpha-1}\left(1+\sup _{0 \leq t \leq T}\left\|\mu_{t}\right\|_{\gamma}^{2}\right)+\int_{0}^{t}(t-s)^{2 \alpha-2} E|X(s)|^{2} \mathrm{~d} s\right] \\
& \leq \frac{k T^{2 \alpha}}{\Gamma(\alpha)^{2}(2 \alpha-1)}\left(1+\sup _{0 \leq t \leq T}\left\|\mu_{t}\right\|_{\gamma}^{2}\right)+\frac{T k}{\Gamma(\alpha)^{2}} \int_{0}^{t}(t-s)^{2 \alpha-2} E|X(s)|^{2} \mathrm{~d} s .
\end{aligned}
$$

For $I_{3}$, by It $\widehat{o}$ 's isometry formula and $\mathrm{H} 2$, we have

$$
\begin{aligned}
I_{3} \leq & \frac{k}{\Gamma(\alpha)^{2}} \int_{0}^{t}(t-s)^{2 \alpha-2}\left(1+E|X(s)|^{2}+\left\|\mu_{t}\right\|_{\gamma}^{2}\right) \mathrm{d} s \\
\leq & \frac{k T^{2 \alpha-1}}{\Gamma(\alpha)^{2}(2 \alpha-1)}\left(1+\sup _{0 \leq t \leq T}\left\|\mu_{t}\right\|_{\gamma}^{2}\right) \\
& +\frac{k}{\Gamma(\alpha)^{2}} \int_{0}^{t}(t-s)^{2 \alpha-2} E|X(s)|^{2} \mathrm{~d} s .
\end{aligned}
$$

Combining the above estimate results, we finally obtain

$$
E|X(t)|^{2} \leq r_{1}+r_{2} \int_{0}^{t}(t-s)^{(2 \alpha-1)-1} E|X(s)|^{2} \mathrm{~d} s,
$$

where

$$
\begin{aligned}
& r_{1}:=3 E\left|x_{0}\right|^{2}+3 \frac{\left(k T^{2 \alpha-1}\right)(T+1)}{\Gamma(\alpha)^{2}(2 \alpha-1)}\left(1+\sup _{0 \leq t \leq T}\left|\mu_{t}\right|_{\gamma}^{2}\right), \\
& r_{2}:=3 \frac{k(T+1)}{\Gamma(\alpha)^{2}} .
\end{aligned}
$$

With the help of Lemma 1, it follows

$$
\begin{aligned}
E|X(t)|^{2} & \leq r_{1}\left(1+\int_{0}^{t} \sum_{n=1}^{\infty} \frac{\left(r_{2} \Gamma(2 \alpha-1)\right)^{n}}{\Gamma((2 \alpha-1) n)}(t-s)^{(2 \alpha-1) n-1} \mathrm{~d} s\right) \\
& \leq r_{1}\left(1+\sum_{n=1}^{\infty} \frac{\left(r_{2} \Gamma(2 \alpha-1) T^{2 \alpha-1}\right)^{n}}{\Gamma((2 \alpha-1) n+1)}\right) \\
& =r_{1}\left(1+E_{2 \alpha-1,1}\left(r_{2} \Gamma(2 \alpha-1) T^{2 \alpha-1}\right)\right)<\infty,
\end{aligned}
$$

for $\forall t \in[0, T]$, and $E_{2 \alpha-1,1}(\cdot)$ is a two-parameter function of the Mittag-Leffler type [21].

Then,

$$
\sup _{0 \leq t \leq T} E|X(t)|^{2}<\infty
$$

and $X_{t} \in L^{\infty}\left([0, T], L^{2}(\Omega ; H)\right)$.

(ii) Now, we show that $X_{t} \in C\left([0, T], L^{2}(\Omega ; H)\right)$ for $\forall \mu \in C\left([0, T],\left(M_{\gamma^{2}}(H), \rho\right)\right):$ 


$$
\begin{aligned}
E\left\|X_{t}-X_{t_{0}}\right\|^{2} \leq & 2 E \frac{1}{\Gamma(\alpha)^{2}}\left\|\int_{0}^{t}(t-s)^{\alpha-1} f\left(s, X_{s}, \mu_{s}\right) \mathrm{d} s-\int_{0}^{t_{0}}\left(t_{0}-s\right)^{\alpha-1} f\left(s, X_{s}, \mu_{s}\right) \mathrm{d} s\right\|^{2} \\
& +2 E \frac{1}{\Gamma(\alpha)^{2}}\left\|\int_{0}^{t}(t-s)^{\alpha-1} f\left(s, X_{s}, \mu_{s}\right) \mathrm{d} B_{s}-\int_{0}^{t_{0}}\left(t_{0}-s\right)^{\alpha-1} f\left(s, X_{s}, \mu_{s}\right) \mathrm{d} B_{s}\right\|^{2} \\
= & 2\left(J_{1}+J_{2}\right) .
\end{aligned}
$$

For $J_{1}$, we have

$$
\begin{aligned}
J_{1} \leq & 2 E \frac{1}{\Gamma(\alpha)^{2}}\left\|\int_{t_{0}}^{t}(t-s)^{\alpha-1} f\left(s, X_{s}, \mu_{s}\right) \mathrm{d} s\right\|^{2} \\
& +2 E \frac{1}{\Gamma(\alpha)^{2}}\left\|\int_{0}^{t_{0}}\left((t-s)^{\alpha-1}-\left(t_{0}-s\right)^{\alpha-1}\right) f\left(s, X_{s}, \mu_{s}\right) \mathrm{d} s\right\|^{2}=: 2 J_{11}+2 J_{12} .
\end{aligned}
$$

By the Cauchy-Schwartz inequality,

For $J_{12}$, we have

$$
\begin{aligned}
J_{11} & \leq \frac{1}{\Gamma(\alpha)^{2}} k \int_{t_{0}}^{t}(t-s)^{2 \alpha-2} \mathrm{~d} s \int_{t_{0}}^{t} E\left|f\left(s, X_{s}, \mu_{s}\right)\right|^{2} \mathrm{~d} s \\
& \leq \frac{k}{\Gamma(\alpha)^{2}(2 \alpha-1)}\left(t-t_{0}\right)^{2 \alpha-1} \int_{t_{0}}^{t}\left(1+E\left|X_{s}\right|^{2}+\left\|\mu_{s}\right\|^{2}\right) \mathrm{d} s \\
& \leq \frac{C k}{\Gamma(\alpha)^{2}(2 \alpha-1)}\left(t-t_{0}\right)^{2 \alpha} .
\end{aligned}
$$

$$
\begin{aligned}
J_{12} & =E \frac{1}{\Gamma(\alpha)^{2}}\left|\int_{0}^{t_{0}}\left((t-s)^{\alpha-1}-\left(t_{0}-s\right)^{\alpha-1}\right) f\left(s, X_{s}, \mu_{s}\right) \mathrm{d} s\right|^{2} \\
& \leq \frac{k}{\Gamma(\alpha)^{2}} \int_{0}^{t_{0}}\left((t-s)^{\alpha-1}-\left(t_{0}-s\right)^{\alpha-1}\right)^{2} \mathrm{~d} s \int_{0}^{t_{0}}\left(1+E\left|X_{s}\right|^{2}+\left\|\mu_{s}\right\|^{2}\right) \mathrm{d} s \\
& \leq \frac{C T k}{\Gamma(\alpha)^{2}} \int_{0}^{t_{0}}\left(\left(t_{0}-s\right)^{2(\alpha-1)}-(t-s)^{2(\alpha-1)}\right) \mathrm{d} s \\
& \leq \frac{C T k}{\Gamma(\alpha)^{2}}\left[\frac{\left(t-t_{0}\right)^{2 \alpha-1}}{2 \alpha-1}+\frac{t_{0}^{2 \alpha-1}}{2 \alpha-1}-\frac{t^{2 \alpha-1}}{2 \alpha-1}\right] \leq \frac{C T k}{\Gamma(\alpha)^{2}} \frac{\left(t-t_{0}\right)^{2 \alpha-1}}{2 \alpha-1} .
\end{aligned}
$$

For $J_{2}$, using the It $\widehat{o}$ isometry formula, in the similar way as $J_{1}$, we can prove that

$$
J_{2} \leq \frac{C k}{\Gamma(\alpha)^{2}} \frac{\left(t-t_{0}\right)^{2 \alpha-1}}{2 \alpha-1}
$$

Results of $J_{1}$ and $J_{2}$ combined together show that

$$
\begin{aligned}
E\left\|X_{t}-X_{t_{0}}\right\|^{2} \leq & \frac{C k(1+T)}{\Gamma(\alpha)^{2}} \frac{\left(t-t_{0}\right)^{2 \alpha-1}}{2 \alpha-1} \\
& +\frac{C k}{\Gamma(\alpha)^{2}(2 \alpha-1)}\left(t-t_{0}\right)^{2 \alpha},
\end{aligned}
$$

which implied $X_{t} \in C\left([0, T], L^{2}(\Omega ; H)\right)$ for each fixed $\mu \in C\left([0, T],\left(M_{\gamma^{2}}, \rho\right)\right)$. 
(iii) By virtue of the fixed point theorem for contraction mappings, we can show that, for each fixed $\mu \in C\left([0, T],\left(M_{\gamma^{2}}, \rho\right)\right)$, equation (10) has a unique solution in $C\left([0, T], L^{2}(\Omega ; H)\right)$. Similar arguments are also discussed in [18]. Now, we define an operator $\Phi_{\mu}(\cdot)$ on $C\left([0, T], L^{2}(\Omega ; H)\right)$ :

$$
\begin{aligned}
\left(\Phi_{\mu} X\right)(t)= & x_{0}+\frac{1}{\Gamma(\alpha)} \int_{0}^{t}(t-s)^{\alpha-1} f\left(s, X_{s}, \mu_{s}\right) \mathrm{d} s \\
& +\frac{1}{\Gamma(\alpha)} \int_{0}^{t}(t-s)^{\alpha-1} g\left(t, X_{s}, \mu_{s}\right) \mathrm{d} B_{t} .
\end{aligned}
$$

It is easy to verify that $\Phi_{\mu}$ is from $C\left([0, T], L^{2}(\Omega ; H)\right)$ into itself.

For $X_{t}, Y_{t} \in C\left([0, T], L^{2}(\Omega ; H)\right)$ with $x_{0}=y_{0}$, let $|\cdot|_{\zeta}$ denote the norm of $C\left([0, T], L^{2}(\Omega ; H)\right)$ and $\beta=2 \alpha-1>0$; then, we obtain

$$
\begin{aligned}
& \left|\left(\Phi_{\mu} X\right)(t)-\left(\Phi_{\mu} Y\right)(t)\right|_{\varsigma}^{2} \\
& \leq 2\left|\frac{1}{\Gamma(\alpha)} \int_{0}^{t}(t-s)^{\alpha-1}\left(f\left(s, X_{s}, \mu_{s}\right)-f\left(s, Y_{s}, \mu_{s}\right)\right) \mathrm{d} s\right|_{\varsigma}^{2} \\
& \quad+2\left|\frac{1}{\Gamma(\alpha)} \int_{0}^{t}(t-s)^{\alpha-1}\left(g\left(t, X_{s}, \mu_{s}\right)-g\left(t, Y_{s}, \mu_{s}\right)\right) \mathrm{d} B_{t}\right|_{\varsigma}^{2}
\end{aligned}
$$

Using the Cauchy-Schwartz inequality and It $\widehat{o}$ isometry formula, it is readily seen that

$$
\begin{aligned}
& \left|\left(\Phi_{\mu} X\right)(t)-\left(\Phi_{\mu} Y\right)(t)\right|_{\varsigma}^{2} \\
& \quad \leq \frac{2 T k+2 k}{\Gamma(\alpha)^{2}} \int_{0}^{t}(t-s)^{\beta-1}\left|X_{s}-Y_{s}\right|_{\varsigma}^{2} \mathrm{~d} s .
\end{aligned}
$$

Our goal is now to prove the following inequality:

$$
\begin{aligned}
& \left(\Phi_{\mu}^{n} X\right)(t)-\left.\left(\Phi_{\mu}^{n} X\right)(t)\right|_{\varsigma} ^{2} \\
& \quad \leq \frac{1}{\beta}\left(\frac{2 T k+2 k}{\Gamma(\alpha)^{2}}\right) \frac{\Gamma(\beta)^{n}}{\Gamma(n \beta)} t^{n \beta}\left|X_{t}-Y_{t}\right|_{\varsigma}^{2} .
\end{aligned}
$$

The proof is based on mathematical induction over $n$. For $n=1$,

$$
\begin{gathered}
\left|\left(\Phi_{\mu} X\right)(t)-\left(\Phi_{\mu} Y\right)(t)\right|_{\varsigma}^{2} \\
\leq \frac{2 T k+2 k}{\Gamma(\alpha)^{2}}\left|X_{t}-Y_{t}\right|_{\varsigma} t^{t^{\beta}},
\end{gathered}
$$

which is fulfilled.

For the induction step from $n=l$ to $n=l+1$, we assume that, for $n=l$, equation (29) is satisfied; then,

$$
\begin{aligned}
& \left|\left(\Phi_{\mu}^{l+1} X\right)(t)-\left(\Phi_{\mu}^{l+1} Y\right)(t)\right|_{\varsigma}^{2} \\
& \leq \frac{2 T k+2 k}{\Gamma(\alpha)^{2}} \int_{0}^{t}(t-s)^{\beta-1}\left|\left(\Phi_{\mu}^{l} X\right)(s)-\left(\Phi_{\mu}^{l} Y\right)(s)\right|_{\varsigma}^{2} \mathrm{~d} s \\
& \leq \frac{2 T k+2 k}{\Gamma(\alpha)^{2}} \int_{0}^{t}(t-s)^{\beta-1} \frac{1}{\beta}\left(\frac{2 T k+2 k}{\Gamma(\alpha)^{2}}\right)^{l} \frac{\Gamma(\beta)^{l}}{\Gamma(k \beta)} s^{l \beta}\left|X_{s}-Y_{s}\right|_{\zeta}^{2} \mathrm{~d} s \\
& \leq\left(\frac{2 T k+2 k}{\Gamma(\alpha)^{2}}\right)^{l+1} \frac{1}{\beta} \frac{\Gamma(\beta)^{k}}{\Gamma(l \beta)}\left|X_{t}-Y_{t}\right|_{\zeta}^{2} \int_{0}^{t}(t-s)^{\beta-1} s^{l \beta} \mathrm{d} s .
\end{aligned}
$$

Thus, we only need to discuss the following integral:

$$
\int_{0}^{t}(t-s)^{\beta-1} s^{l \beta} \mathrm{d} s
$$

Let $s=t z$; then,

$$
\begin{aligned}
\int_{0}^{t}(t-s)^{\beta-1} s^{l \beta} \mathrm{d} s & =\int_{0}^{1}(1-z)^{\beta-1} t^{\beta-1} t^{l \beta} z^{l \beta} \mathrm{d} z \\
& =t^{(l+1) \beta} \int_{0}^{1}(1-z)^{\beta-1} z^{l \beta} \mathrm{d} z \\
& =t^{(l+1) \beta} B(l \beta+1, \beta)=t^{(l+1) \beta} \frac{\Gamma(\beta) \Gamma(l \beta+1)}{\Gamma((l+1) \beta+1)},
\end{aligned}
$$

where $B(\cdot, \cdot)$ is the Beta function. Substitute the above equality into (31), and we derive that 


$$
\begin{aligned}
& \left|\left(\Phi_{\mu}^{l+1} X\right)(t)-\left(\Phi_{\mu}^{l+1} Y\right)(t)\right|_{\varsigma}^{2} \\
& \leq\left(\frac{2 T k+2 k}{\Gamma^{2}(\alpha)}\right)^{l+1} \frac{1}{\beta} \frac{\Gamma(\beta)}{\Gamma(l \beta)}\left|X_{t}-Y_{t}\right|_{\varsigma}^{2} t^{(l+1) \beta} \frac{\Gamma(\beta) \Gamma(l \beta+1)}{\Gamma((l+1) \beta+1)} \\
& =\left(\frac{2 T k+2 k}{\Gamma^{2}(\alpha)}\right)^{l+1} \frac{1}{\beta} \Gamma(\beta)^{l+1} \frac{\Gamma(l \beta+1)}{\Gamma((l+1) \beta+1) \Gamma(l \beta)} t^{(l+1) \beta}\left|X_{t}-Y_{t}\right|_{\varsigma}^{2} \\
& =\left(\frac{2 T k+2 k}{\Gamma^{2}(\alpha)}\right)^{l+1} \frac{1}{\beta} \Gamma(\beta)^{l+1} \frac{l \beta \Gamma(l \beta)}{(l+1) \beta \Gamma((l+1) \beta) \Gamma(l \beta)} t^{(l+1) \beta}\left|X_{t}-Y_{t}\right|_{\varsigma}^{2} \\
& \leq\left(\frac{2 T k+2 k}{\Gamma^{2}(\alpha)}\right)^{l+1} \frac{1}{\beta} \Gamma(\beta)^{l+1} \frac{t^{(l+1) \beta}}{\Gamma((l+1) \beta)}\left|X_{t}-Y_{t}\right|_{\varsigma}^{2} \cdot
\end{aligned}
$$

By the above discussion, we finally obtain

$$
\begin{aligned}
& \left|\left(\Phi_{\mu}^{n} X\right)(t)-\left(\Phi_{\mu}^{n} Y\right)(t)\right|_{\varsigma}^{2} \\
& \quad \leq\left(\frac{2 T k+2 k}{\Gamma^{2}(\alpha)}\right)^{n} \frac{1}{\beta} \Gamma(\beta)^{n} \frac{T^{n \beta}}{\Gamma(n \beta)}\left|X_{t}-Y_{t}\right|_{\varsigma}^{2} .
\end{aligned}
$$

Note that $(T k+k / \Gamma(\alpha))^{n}(1 / \beta) \Gamma(\beta)^{n}\left(T^{n \beta} / \Gamma(n \beta)\right)$ $\longrightarrow 0$ as $n \longrightarrow+\infty$. Then, we have $(T k+k / \Gamma(\alpha))^{n}(1 / \beta) \Gamma(\beta)^{n}\left(T^{n \beta} / \Gamma(n \beta)\right)<1$ for any sufficiently large $n$. And, this shows that $\Phi_{\mu}(\cdot)$ is a contraction map on $C\left([0, T], L^{2}(\Omega ; H)\right)$. So, it has a unique fixed point for $\mu \in C\left([0, T],\left(M_{\gamma^{2}}(H), \rho\right)\right)$.

(iv) $L\left(X_{\mu}\right)=\left\{L\left(X_{\mu}(t)\right): t \in[0, T]\right\}$ is the probability law of $X_{\mu}$. Now, we prove that $L\left(X_{\mu}\right)$ $\in C\left([0, T],\left(M_{\gamma^{2}}, \rho\right)\right)$. Notice that $X_{\mu} \in C([0, T]$, $\left.L^{2}(\Omega ; H)\right), L\left(X_{\mu}\right) \in M_{\gamma^{2}}(H)$ for $\forall t \in[0, T]$. So, we only need to prove $t \longrightarrow L\left(X_{\mu}(t)\right)$ is continuous. In step (ii), we have

$$
E\left|X_{\mu}(t)-X_{\mu}(s)\right|^{2} \longrightarrow 0
$$

as $t \longrightarrow s$.

By the definition of $\rho$, we have

$$
\begin{aligned}
& \left|\left(\phi, L\left(X_{\mu}(t)\right)-L\left(X_{\mu}(s)\right)\right)\right|=E\left|\phi\left(X_{\mu}(t)\right)-\phi\left(X_{\mu}(s)\right)\right| \\
& \leq\|\phi\|_{\rho} E\left|X_{\mu}(t)-X_{\mu}(s)\right| \leq\|\phi\|_{\rho}\left(E\left|X_{\mu}(t)-X_{\mu}(s)\right|^{2}\right)^{1 / 2},
\end{aligned}
$$

which implies

$$
\lim _{t \longrightarrow s} \rho\left(L\left(X_{\mu}(t)\right), L\left(X_{\mu}(s)\right)\right)=0 .
$$

Hence, we verify that $L\left(X_{\mu}\right) \in C\left([0, T],\left(M_{\gamma^{2}}, \rho\right)\right)$. (v) Define $\Psi$ on $C\left([0, T],\left(M_{\gamma^{2}}, \rho\right)\right)$ as follows:

$$
\Psi: \mu \longrightarrow L\left(X_{\mu}\right)
$$

In the following, we will show that the operator $\Psi$ has a unique fixed point in $C\left([0, T],\left(M_{\gamma^{2}}, \rho\right)\right)$. Take $\mu, \nu \in C\left([0, T],\left(M_{\gamma^{2}}, \rho\right)\right)$, and let $X_{\mu}(t)$ and $X_{\nu}(t)$ be the corresponding solutions of the following equations:

$$
\begin{aligned}
X_{\mu}(t)= & x_{0}+\frac{1}{\Gamma(\alpha)} \int_{0}^{t}(t-s)^{\alpha-1} f\left(s, X_{s}, \mu_{s}\right) \mathrm{d} s \\
& +\frac{1}{\Gamma(\alpha)} \int_{0}^{t}(t-s)^{\alpha-1} g\left(t, X_{s}, \mu_{s}\right) \mathrm{d} B_{t}, \\
X_{\nu}(t)= & x_{0}+\frac{1}{\Gamma(\alpha)} \int_{0}^{t}(t-s)^{\alpha-1} f\left(s, X_{s}, v_{s}\right) \mathrm{d} s \\
& +\frac{1}{\Gamma(\alpha)} \int_{0}^{t}(t-s)^{\alpha-1} g\left(t, X_{s}, v_{s}\right) \mathrm{d} B_{t} .
\end{aligned}
$$

Thus, 


$$
\begin{aligned}
E\left|X_{\mu}(t)-X_{v}(t)\right|^{2} \leq & \frac{2 T}{\Gamma(\alpha)^{2}} E \int_{0}^{t}(t-s)^{2 \alpha-2}\left|f\left(s, X_{\mu}(s), \mu_{s}\right)-f\left(s, X_{v}(s), v_{s}\right)\right|^{2} \mathrm{~d} s \\
& +\frac{2}{\Gamma(\alpha)^{2}} E \int_{0}^{t}(t-s)^{2 \alpha-2}\left|g\left(s, X_{\mu}(s), \mu_{s}\right)-g\left(s, X_{v}(s), v_{s}\right)\right|^{2} \mathrm{~d} s \\
\leq & \frac{2 k T}{\Gamma(\alpha)^{2}} E \int_{0}^{t}(t-s)^{2 \alpha-2}\left[\left|X_{\mu}(s)-X_{v}(s)\right|^{2}+\rho^{2}\left(\mu_{s}, v_{s}\right)\right] \mathrm{d} s \\
& +\frac{2 k}{\Gamma(\alpha)^{2}} E \int_{0}^{t}(t-s)^{2 \alpha-2}\left[\left|X_{\mu}(s)-X_{v}(s)\right|^{2}+\rho^{2}\left(\mu_{s}, v_{s}\right)\right] \mathrm{d} s .
\end{aligned}
$$

After simple calculation, we have that

$$
\sup _{0 \leq t \leq T} E\left|X_{\mu}(t)-X_{\nu}(t)\right|^{2} \leq \frac{2 k(T+1)}{\Gamma(\alpha)^{2}} \frac{T^{2 \alpha-1}}{2 \alpha-1}\left[\sup _{0 \leq t \leq T} E\left|X_{\mu}(t)-X_{\nu}(t)\right|^{2}+D_{T}^{2}(\mu, \nu)\right]
$$

Select the appropriate $T=T_{0}>0$, such that

$$
\frac{2 k\left(T_{0}+1\right)}{\Gamma(\alpha)^{2}} \frac{T_{0}^{2 \alpha-1}}{2 \alpha-1}<\frac{1}{3} \text {. }
$$

Then, it follows

$$
\sup _{0 \leq t \leq T_{0}} E\left|X_{\mu}(t)-X_{\nu}(t)\right|^{2}<\frac{1}{2} D_{T_{0}}^{2}(\mu, v) .
$$

By the definition of $\rho(\mu, \nu)$ and $D_{T}^{2}(\mu, \nu)$, we can obtain

$$
\rho^{2}(\mu, \nu) \leq E\left|X_{\mu}(t)-X_{\nu}(t)\right|^{2}
$$

Taking sup-norm on both sides, we obtain

$$
D_{T}^{2}(\Psi(\mu), \Psi(\nu)) \leq \sup _{0 \leq t \leq T_{0}} E\left|X_{\mu}(t)-X_{\nu}(t)\right|^{2} .
$$

Combine this result with equation (44), and we finally derive

$$
D_{T_{0}}^{2}(\Psi(\mu), \Psi(\nu))<\frac{1}{2} D_{T_{0}}^{2}(\mu, \nu) .
$$

Since $\Psi$ is a contraction in $C\left(\left[0, T_{0}\right],\left(M_{\gamma^{2}}(H), \rho\right)\right)$, it has a unique fixed point. Thus, equation (10) has a unique solution $X_{t}$ with $\mu=L\left(X_{t}\right)$ on $\left[0, T_{0}\right]$. Because $X_{t}$ belongs to $C\left([0, T], L^{2}(\Omega ; H)\right)$, we can extend the solution to $[0, T]$ by considering $\left[0, T_{0}\right],\left[T_{0}, 2 T_{0}\right]$, and so on. This completes the proof.

\section{An Averaging Principle}

In this section, we study an averaging principle for the following distribution dependent fractional stochastic differential equations in $H$ :

$$
\begin{aligned}
X_{\epsilon}(t)= & x_{0}+\frac{\epsilon}{\Gamma(\alpha)} \int_{0}^{t}(t-s)^{\alpha-1} f\left(s, X_{\epsilon}(s), \mu_{\epsilon}(s)\right) \mathrm{d} s \\
& +\frac{\sqrt{\epsilon}}{\Gamma(\alpha)} \int_{0}^{t}(t-s)^{\alpha-1} g\left(s, X_{\epsilon}(s), \mu_{\epsilon}(s)\right) \mathrm{d} B_{t},
\end{aligned}
$$

where $x_{0} \in L^{2}(\Omega ; H)$. We will show that the solution of (48) will be approximated by the following simpler or averaged process under certain conditions:

$$
\begin{aligned}
Z_{\epsilon}(t)= & x_{0}+\frac{\epsilon}{\Gamma(\alpha)} \int_{0}^{t}(t-s)^{\alpha-1} \bar{f}\left(Z_{\epsilon}(s), v_{\epsilon}(s)\right) \mathrm{d} s \\
& +\frac{\sqrt{\epsilon}}{\Gamma(\alpha)} \int_{0}^{t}(t-s)^{\alpha-1} \bar{g}\left(Z_{\epsilon}(s), v_{\epsilon}(s)\right) \mathrm{d} B_{t} .
\end{aligned}
$$

Equation (49) is called the averaged equation for (48). Now, we prove that the solution of (49) converges to the solution of the original equation (48) under the following additional conditions.

H3:

$$
\begin{aligned}
& \frac{1}{t^{2 \alpha-1}} \int_{0}^{t}(t-s)^{2(\alpha-1)}|f(s, x, \mu)-\bar{f}(x, \mu)|^{2} \mathrm{~d} s \\
& \quad \leq \varphi_{1}(t)\left(1+|x|^{2}+\|\mu\|^{2}\right) .
\end{aligned}
$$

H4:

$$
\begin{aligned}
& \frac{1}{t^{2 \alpha-1}} \int_{0}^{t}(t-s)^{2(\alpha-1)}\left|g(s, x, \mu)-\bar{g}(x, \mu)^{2}\right| \mathrm{d} s \\
& \quad \leq \varphi_{2}(t)\left(1+|x|^{2}+\|\mu\|^{2}\right),
\end{aligned}
$$


where $\varphi_{i}(t)$ are positive and bounded with $\lim _{t \longrightarrow+\infty} \varphi_{i}(t)=0$ for $i=1,2$.

Remark 1. Note that when we take $\alpha=1$, then this condition is consistence with the classic case, see [4].

Let us consider

Theorem 2. Let $H 1-H 4$ hold. Then, for $\forall \delta_{1}>0$, there exist constants $L>0, \epsilon_{1} \in\left(0, \epsilon_{0}\right]$ and $\beta \in(0,1)$ such that, for any $\epsilon \in\left(0, \epsilon_{1}\right], 1 / 2<\alpha<1$, we have

$$
\sup _{0 \leq t \leq L \mathcal{E}^{(-\beta / 2 \alpha-1)}} E\left|X_{\varepsilon}(t)-Z_{\varepsilon}(t)\right|^{2} \leq \delta_{1} .
$$

$$
\begin{aligned}
X_{\epsilon}(t)-Z_{\epsilon}(t)= & \frac{\epsilon}{\Gamma(\alpha)} \int_{0}^{t}(t-s)^{\alpha-1}\left[f\left(s, X_{\epsilon}(s), \mu_{\epsilon}(s)\right)-\bar{f}\left(Z_{\epsilon}(s), v_{\epsilon}(s)\right)\right] \mathrm{d} s \\
& +\frac{\sqrt{\epsilon}}{\Gamma(\alpha)} \int_{0}^{t}(t-s)^{\alpha-1}\left[g\left(s, X_{\epsilon}(s), \mu_{\epsilon}(s)\right)-\bar{g}\left(Z_{\epsilon}(s), v_{\epsilon}(s)\right)\right] \mathrm{d} B_{t} .
\end{aligned}
$$

By the arithmetic inequality, it follows that

$$
\begin{aligned}
& E\left|X_{\epsilon}(t)-Z_{\epsilon}(t)\right|^{2} \leq \\
& 2\left\{\begin{array}{c}
E\left|\frac{\epsilon}{\Gamma(\alpha)} \int_{0}^{t}(t-s)^{\alpha-1}\left[f\left(s, X_{\epsilon}(s), \mu_{\epsilon}(s)\right)-\bar{f}\left(Z_{\epsilon}(s), v_{\epsilon}(s)\right)\right] \mathrm{d} s\right|^{2} \\
+E\left|\frac{\sqrt{\epsilon}}{\Gamma(\alpha)} \int_{0}^{t}(t-s)^{\alpha-1}\left[g\left(s, X_{\epsilon}(s), \mu_{\epsilon}(s)\right)-\bar{g}\left(Z_{\epsilon}(s), v_{\epsilon}(s)\right)\right] \mathrm{d} B_{t}\right|^{2}
\end{array}\right\} \\
& =: 2\left(Q_{1}+Q_{2}\right) .
\end{aligned}
$$

For $Q_{1}$, we have

$$
\begin{aligned}
Q_{1} & =E\left|\frac{\epsilon}{\Gamma(\alpha)} \int_{0}^{t}(t-s)^{\alpha-1}\left[f\left(s, X_{\epsilon}(s), \mu_{\epsilon}(s)\right)-\bar{f}\left(Z_{\epsilon}(s), v_{\epsilon}(s)\right)\right] \mathrm{d} s\right|^{2} \\
& \leq \frac{2 \epsilon^{2}}{\Gamma(\alpha)^{2}}\left\{\begin{array}{c}
E\left|\int_{0}^{t}(t-s)^{\alpha-1}\left[f\left(s, X_{\epsilon}(s), \mu_{\epsilon}(s)\right)-f\left(s, Z_{\epsilon}(s), v_{\epsilon}(s)\right)\right] \mathrm{d} s\right|^{2} \\
+E\left|\int_{0}^{t}(t-s)^{\alpha-1}\left[f\left(s, Z_{\epsilon}(s), v_{\epsilon}(s)\right)-\bar{f}\left(Z_{\epsilon}(s), v_{\epsilon}(s)\right)\right] \mathrm{d} s\right|^{2}
\end{array}\right\} .
\end{aligned}
$$


Applying the Cauchy-Schwarz inequality, H1, H3, and the definition of the metric $\rho$, we obtain

$$
\begin{aligned}
& Q_{1} \leq \frac{2 t k \epsilon^{2}}{\Gamma(\alpha)^{2}} \int_{0}^{t}(t-s)^{2 \alpha-2} E\left[\left|X_{\epsilon}(s)-Z_{\epsilon}(s)\right|^{2}+\rho^{2}\left(\mu_{\epsilon}(s), \nu_{\epsilon}(s)\right)\right] \mathrm{d} s \\
& +\frac{2 \epsilon^{2}}{\Gamma(\alpha)^{2}} t^{2 \alpha}\left\{\begin{array}{c}
\frac{1}{t^{2 \alpha-1}} E \int_{0}^{t}(t-s)^{2 \alpha-2}\left|f\left(s, Z_{\epsilon}(s), \nu_{\epsilon}(s)\right)-\bar{f}\left(Z_{\epsilon}(s), v_{\epsilon}(s)\right)\right|^{2} \mathrm{~d} s \\
\leq \frac{4 t k \epsilon^{2}}{\Gamma(\alpha)^{2}} \int_{0}^{t}(t-s)^{2 \alpha-2} E\left|X_{\epsilon}(s)-Z_{\epsilon}(s)\right|^{2} \mathrm{~d} s \\
+\frac{2 \epsilon^{2}}{\Gamma(\alpha)^{2}} t^{2 \alpha} \varphi_{1}(t)\left(1+E\left|Z_{\epsilon}(s)\right|^{2}+\left\|\nu_{\epsilon}(s)\right\|\right)^{2}
\end{array}\right\} .
\end{aligned}
$$

For $Q_{2}$, using the Itô isometry formula, we obtain

$$
\begin{aligned}
Q_{2} & =\frac{\epsilon}{\Gamma(\alpha)^{2}} \int_{0}^{t}(t-s)^{2 \alpha-2} E\left|g\left(s, X_{\epsilon}(s), \mu_{\epsilon}(s)\right)-\bar{g}\left(Z_{\epsilon}(s), v_{\epsilon}(s)\right)\right|^{2} \mathrm{~d} s \\
& \leq \frac{2 \epsilon}{\Gamma(\alpha)^{2}}\left\{\begin{array}{l}
\int_{0}^{t}(t-s)^{2 \alpha-2} E\left|g\left(s, X_{\epsilon}(s), \mu_{\epsilon}(s)\right)-g\left(Z_{\epsilon}(s), \nu_{\epsilon}(s)\right)\right|^{2} \mathrm{~d} s \\
+\int_{0}^{t}(t-s)^{2 \alpha-2} E\left|g\left(Z_{\epsilon}(s), v_{\epsilon}(s)\right)-\bar{g}\left(Z_{\epsilon}(s), v_{\epsilon}(s)\right)\right|^{2} \mathrm{~d} s
\end{array}\right\} .
\end{aligned}
$$

Applying conditions $\mathrm{H} 1$ and $\mathrm{H} 4$, we derive

$$
\begin{aligned}
Q_{2} \leq & \frac{2 k \epsilon}{\Gamma(\alpha)^{2}}\left\{\begin{array}{l}
\int_{0}^{t}(t-s)^{2(\alpha-1)}\left[E\left|X_{\epsilon}(s)-Z_{\epsilon}(s)\right|^{2}+\rho^{2}\left(\mu_{\epsilon}(s), v_{\epsilon}(s)\right)\right] \mathrm{d} s \\
+\int_{0}^{t}(t-s)^{2(\alpha-1)} E\left|g\left(Z_{\epsilon}(s), \nu_{\epsilon}(s)\right)-\bar{g}\left(Z_{\epsilon}(s), v_{\epsilon}(s)\right)\right|^{2} \mathrm{~d} s
\end{array}\right\} \\
\leq & \frac{4 k \epsilon}{\Gamma(\alpha)^{2}} \int_{0}^{t}(t-s)^{2(\alpha-1)} E\left|X_{\epsilon}(s)-Z_{\epsilon}(s)\right|^{2} \mathrm{~d} s \\
& +\frac{2 \epsilon}{\Gamma(\alpha)^{2}} t^{2 \alpha-1} \varphi_{2}(t)\left(1+E\left|Z_{\epsilon}(s)\right|^{2}+\left\|\nu_{\epsilon}(s)\right\|^{2}\right) .
\end{aligned}
$$


Therefore, from the above discussion, (56)-(58), and Theorem 1, we have

$$
\begin{aligned}
E\left|X_{\epsilon}(t)-Z_{\epsilon}(t)\right|^{2} \leq & \frac{4 t k \epsilon^{2}}{\Gamma(\alpha)^{2}} \int_{0}^{t}(t-s)^{2(\alpha-1)} E\left|X_{\epsilon}(s)-Z_{\epsilon}(s)\right|^{2} \mathrm{~d} s \\
& +\frac{2 \epsilon^{2}}{\Gamma(\alpha)^{2}} t^{2 \alpha} \varphi_{1}\left((t)\left(1+E\left|Z_{\epsilon}(s)\right|^{2}+\left\|\nu_{\epsilon}(s)\right\|^{2}\right)\right) \\
& +\frac{4 k \epsilon}{\Gamma(\alpha)^{2}} \int_{0}^{t}(t-s)^{2(\alpha-1)} E\left|X_{\epsilon}(s)-Z_{\epsilon}(s)\right|^{2} \mathrm{~d} s+\frac{2 \epsilon}{\Gamma(\alpha)^{2}} t^{2 \alpha-1} \varphi_{2} \Gamma(\alpha)^{2}\left(1+E\left|Z_{\epsilon}(s)\right|^{2}+\left\|\nu_{\epsilon}(s)\right\|^{2}\right) \\
= & 2 \epsilon T^{2 \alpha-1}\left(\frac{c_{1} \epsilon}{\Gamma(\alpha)^{2}} T+\frac{c_{2}}{\Gamma(\alpha)^{2}}\right)+4 \epsilon\left(\frac{k \epsilon}{\Gamma(\alpha)^{2}} T+\frac{k}{\Gamma(\alpha)^{2}}\right) \int_{0}^{t}(t-s)^{(2 \alpha-1)-1} E\left|X_{\epsilon}(s)-Z_{\epsilon}(s)\right|^{2} \mathrm{~d} s .
\end{aligned}
$$

Denote $r_{1}=2\left(\left(c_{1} \epsilon / \Gamma(\alpha)^{2}\right) T+\left(c_{2} / \Gamma(\alpha)^{2}\right)\right)$ and $r_{2}=$ $4\left(\left(k \epsilon / \Gamma(\alpha)^{2}\right) T+\left(k / \Gamma(\alpha)^{2}\right)\right)$; using Lemma 1 , we have

$$
\begin{aligned}
& E\left|X_{\epsilon}(t)-Z_{\epsilon}(t)\right|^{2} \leq \epsilon T^{2 \alpha-1} r_{1}\left(1+\int_{0}^{t} \sum_{n=1}^{\infty} \frac{\left(r_{2} \epsilon \Gamma(2 \alpha-1)\right)^{n}}{\Gamma((2 \alpha-1) n)}(t-s)^{(2 \alpha-1) n-1} \mathrm{~d} s\right) \\
& \leq \epsilon T^{2 \alpha-1} r_{1}\left(1+\sum_{n=1}^{\infty} \frac{\left(r_{2} \epsilon \Gamma(2 \alpha-1) T^{2 \alpha-1}\right)^{n}}{\Gamma((2 \alpha-1) n+1)}\right) \\
& \leq \epsilon T^{2 \alpha-1} r_{1}\left(1+E_{2 \alpha-1,1}\left(r_{2} \epsilon \Gamma(2 \alpha-1) T^{2 \alpha-1}\right)\right) . \\
& \quad D_{T}\left(\mu_{\epsilon}, v_{\epsilon}\right)=\sup _{0 \leq t \leq T} \rho\left(\mu_{\epsilon}(t), v_{\epsilon}(t)\right) \\
& \leq \sup _{0 \leq t \leq T} E\left|X_{\epsilon}(t)-Z_{\epsilon}(t)\right|^{2} \longrightarrow 0, \quad \epsilon \longrightarrow 0,
\end{aligned}
$$

Select some $\beta \in(0,1), L>0$, such that, for $\forall t \in(0, L \epsilon$ $(-\beta / 2 \alpha-1)]$, we obtain

$$
\sup _{0 \leq t \leq L \epsilon^{(-\beta / 2 \alpha-1)}} E\left|X_{\epsilon}(t)-Z_{\epsilon}(t)\right|^{2} \leq C \epsilon^{1-\beta}
$$

where $C=r_{1}\left(1+E_{2 \alpha-1,1}\left(r_{2} L \epsilon^{(-\beta / 2 \alpha-1)} \Gamma(2 \alpha-1)\right)\right)$.

Consequently, for $\forall \delta_{1}>0$, one can select some $\epsilon_{1} \in\left(0, \epsilon_{0}\right]$ such that, for each $\epsilon \in\left(0, \epsilon_{1}\right], \forall t \in$ $\left(0, L \epsilon^{(-\beta / 2 \alpha-1)}\right]$, we have

$$
\sup _{0 \leq t \leq L \epsilon}^{(-\beta / 2 \alpha-1)} \in\left|X_{\epsilon}(t)-Z_{\epsilon}(t)\right|^{2} \leq \delta_{1} .
$$

This completes the proof.

Remark 2. Using the definition of $\rho$, we obtain

$$
\rho\left(\mu_{\epsilon}(t), v_{\epsilon}(t)\right) \leq E\left|X_{\epsilon}(t)-Z_{\epsilon}(t)\right| \leq E\left|X_{\epsilon}(t)-Z_{\epsilon}(t)\right|^{2} .
$$

From the above estimate, we actually obtain which means that, as $\epsilon \longrightarrow 0, \mu_{\epsilon}(t)$ corresponding to $X_{\epsilon}(t)$ converges to $\nu_{\epsilon}(t)$ of $Z_{\epsilon}(t)$ in $C\left(\left[0, T ;\left(M_{\gamma}^{2}, \rho\right)\right]\right)$.

\section{Data Availability}

No data were used to support this study.

\section{Conflicts of Interest}

The authors declare that they have no conflicts of interest.

\section{Acknowledgments}

This work was partiality supported by the NNSFs of China (nos. 11901584, 61876192, 11926322, and 11801575), "the Fundamental Research Funds for the Central Universities," 
the South-Central University for Nationalities (nos. CZY20013, CZY20014, CTZ20020, and KTZ20051).

\section{References}

[1] J. Duan, K. Lu, and B. r. Schmalfuss, "Smooth stable and unstable manifolds for stochastic evolutionary equations," Journal of Dynamics and Differential Equations, vol. 16, no. 4, pp. 949-972, 2004.

[2] J. Duan, K. Lu, and B. Schmalfuss, "Invariant manifolds for stochastic partial differential equations," Annals of Probability, vol. 31, no. 4, pp. 2109-2135, 2003.

[3] H. Fu and J. Liu, "Strong convergence in stochastic averaging principle for two time-scales stochastic partial differential equations," Journal of Mathematical Analysis and Applications, vol. 384, no. 1, pp. 70-86, 2011.

[4] Y. Xu, J. Duan, and W. Xu, "An averaging principle for stochastic dynamical systems with Lévy noise," Physica D: Nonlinear Phenomena, vol. 240, no. 17, pp. 1395-1401, 2011.

[5] J. Xu and J. Liu, "An Averaging principle for multivalued stochastic differential equations," Stochastic Analysis and Applications, vol. 32, no. 6, pp. 962-974, 2014.

[6] W. Mao, L. Hu, S. You, and X. Mao, "The averaging method for multivalued SDEs with jumps and non-Lipschitz coefficients," Discrete \& Continuous Dynamical Systems-B, vol. 24, no. 9, p. 4937, 2019.

[7] W. Mao, S. You, X. Wu, and X. Mao, "On the averaging principle for stochastic delay differential equations with jumps," Advances in Difference Equations, vol. 2015, no. 1, pp. 1-19, 2015.

[8] J. Bao, G. Yin, and C. Yuan, "Two-time-scale stochastic partial differential equations driven by -stable noises: averaging principles," Bernoulli, vol. 23, pp. 645-669, 2017.

[9] J. Hu and C. Yuan, "Strong convergence of neutral stochastic functional differential equations with two time-scales," Discrete and Continuous Dynamical Systems-B, vol. 24, no. 11, p. 5831, 2019.

[10] B. Pei, Y. Xu, and J.-L. Wu, "Stochastic averaging for stochastic differential equations driven by fractional Brownian motion and standard Brownian motion," Applied Mathematics Letters, vol. 100, Article ID 106006, 2020.

[11] D. Luo, Q. Zhu, and Z. Luo, "An averaging principle for stochastic fractional differential equations with time-delays," Applied Mathematics Letters, vol. 105, Article ID 106290, 2020.

[12] X. He, S. Han, and J. Tao, "Averaging principle for SDEs of neutral type driven by G-Brownian motion," Stochastics and Dynamics, vol. 19, no. 01, Article ID 1950004, 2019.

[13] W. Xu, W. Xu, and S. Zhang, "The averaging principle for stochastic differential equations with Caputo fractional derivative," Applied Mathematics Letters, vol. 93, pp. 79-84, 2019.

[14] R. Z. Khasminskii, "On the principle of averaging the it $\widehat{o}$ stochastic differential equations," Kibernetika, vol. 4, pp. 260-279, 1968.

[15] L. Wu, S. Liu, L. Yao, S. Yan, and D. Liu, "Grey system model with the fractional order accumulation," Communications in Nonlinear Science and Numerical Simulation, vol. 18, no. 7, pp. 1775-1785, 2013.

[16] C. Yan, L. Wu, L. Liu et al., "Fractional Hausdorff grey model and its properties," Chaos Solitons and Fractals, vol. 138, Article ID 109915, 2020.

[17] J. Wen, C. Wu, R. Zhang, X. Xiao, N. Nv, and Y. Shi, "Rearend collision warning of connected automated vehicles based on a novel stochastic local multivehicle optimal velocity model," Accident Analysis \& Prevention, vol. 148, Article ID 105800, 2020.

[18] T. E. Govindan and N. U. Ahmed, "On yosida approximations of McKean-Vlasov type stochastic evolution equations," Stochastic Analysis and Applications, vol. 33, no. 3, pp. 383398, 2015.

[19] X. Huang and F.-Y. Wang, "Distribution dependent SDEs with singular coefficients," Stochastic Processes and Their Applications, vol. 129, no. 11, pp. 4747-4770, 2019.

[20] F.-Y. Wang, "Distribution dependent SDEs for Landau type equations," Stochastic Processes and Their Applications, vol. 128, no. 2, pp. 595-621, 2018.

[21] Y. Wang, J. Xu, and P. E. Kloeden, “Asymptotic behavior of stochastic lattice systems with a Caputo fractional time derivative," Nonlinear Analysis: Theory, Methods \& Applications, vol. 135, pp. 205-222, 2016.

[22] H. Ye, J. Gao, and Y. Ding, "A generalized Gronwall inequality and its application to a fractional differential equation," Journal of Mathematical Analysis and Applications, vol. 328, no. 2, pp. 1075-1081, 2007. 